Shapes and Textures for Rendering Coral

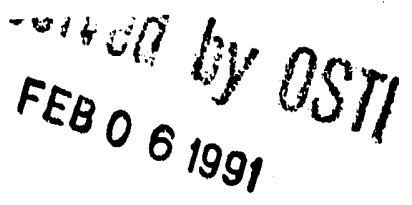

\author{
Nelson L. Max \\ Livermore, California \\ Geoff Wyvill \\ University of Otago \\ New Zealand
}

Lawrence Livermore National Laboratory

This paper was prepared for submittal to

CG International

Cambridge, Massachusetts

June 27 - June 29, 1991

October 18, 1990

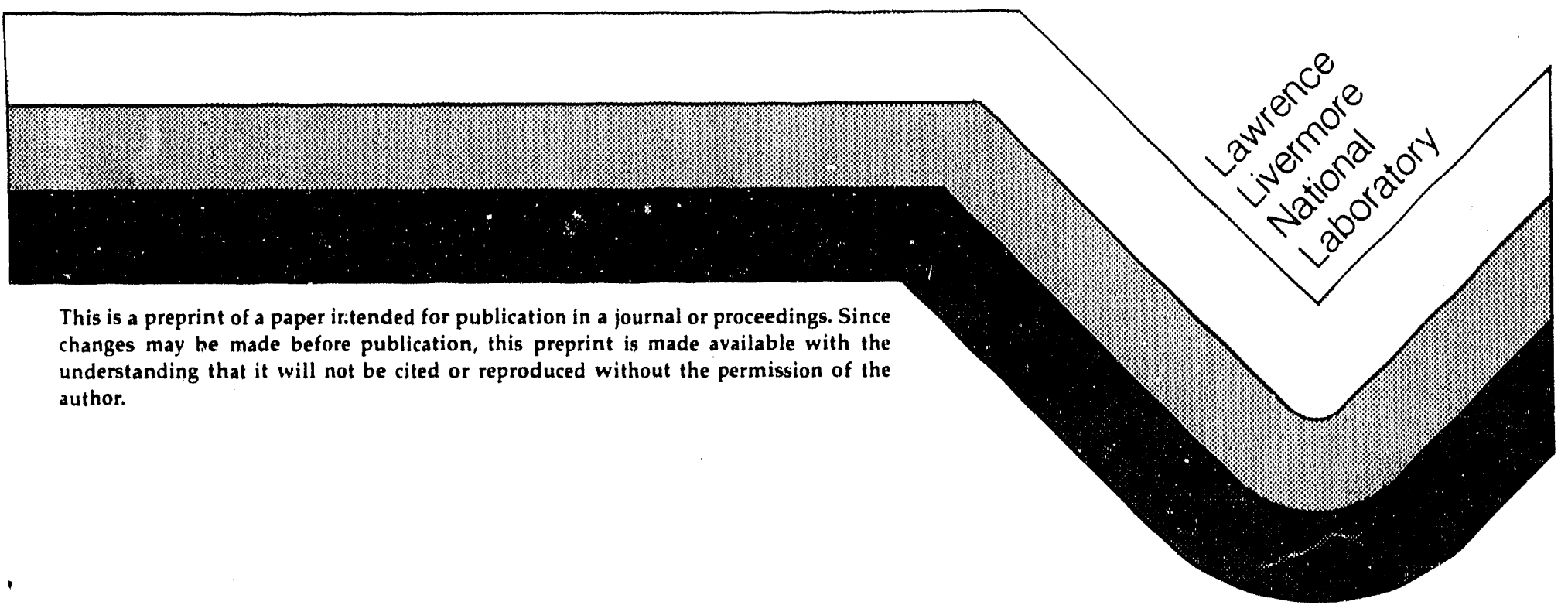


DISCLAINIER

This document was prepared as an account of work sponsored by an agency of the linited Stutes Government. Neither the United States Government nor the University of Californix nor any of their emplovees. makes uny warranty, express or implied, or assumes any lexul liability or responsibility for the accuracy, completeness, or usefulness of any information. apparatus. product. or process disclosed. or represents that its use would not infringe privately owned rights. Reference herein 10 any specific commercial products. process. or service by trade name. trademark. manufacturer, or otherwise. does not necessurily constitute or imply its endorsement. recommendation. or favoring by the United States Guvernment or the Liniversity of California. The views and opinions of authors espressed herein do not necessarily state or reflect those of the Linited States Government or the Liniversity of California. and shall not be used for advertising or product endorsement purposes. 


\title{
Shapes and Textures for Rendering Coral
}

\author{
Nelson Max \\ Geoff Wyvill
}

\begin{abstract}
A growth algorithm has been developed to build coral shapes out of a tree of spheres. A volume density defined by the spheres is contoured to give a "soft object". The resulting contour surfaces are rendered by ray tracing, using a generalized volume texture to produce shading and "bump mapped" normal perturbations.
\end{abstract}

\section{Introduction}

We constructed the coral as a soft object (Wyvill et. al., [1]) inside the Katachi system (Kunii \& Wyvill [2]) and ray traced it (Wyvill \& Trotman [3]). We built the coral by a parametrized growth algorithm, similar to those which have been used to build trees (Bloomenthal [4], Aono \& Kunii [5], De Reffye et.et. al. al. [6], Viennot et. al. [7], and Prusinkiewicz \& Lindenmayer [8]). Kawaguchi [9] has also animated undersea creatures, defined by metaballs (Nishimura et. al. [10]), the Japanese equivalent of soft objects.

The surface of a soft object is the contour of a volume density, defined as the sum of spherically or elliptically symmetric densities centred at various points. In this work, we used only spherical densities, and each was specified by a centre and an outer radius at which the density function becomes zero. Section 2 explains the growth algorithm which governed the placement of these spheres.

We wanted to change the surface colour and perturb the surface normal vector (Blinn [11]) at each point on the coral, in order to render a realistic texture. The standard methods of texture and "bump" mapping look up values in a 2-D colour or bump height table. However such mappings are difficult to apply to a general contour surface, because there is no simple method to assign the co-ordinate functions into the 2-D texture tables. Therefore volume textures (Peachey [12], Perlin [13], Wyvill et. al. [14]) are the preferred method for contour surfaces. These are defined directly as functions of the 3D 
co-ordinates of a surface point, often by analytic computation rather than table look-up. To generate our coral texture, we have generalized this idea, and compute the colour and normal perturbations as functions of both the 3D surface point and the normal to the contour surface. Section 3 describes this generalized volume texture. Section 4 gives our results.

\section{Coral Structure}

The coral structures are "soft objects" contoured from the sum of spherically symmetric density functions, so the structure is determined by the centres of the spheres, and the outer radii at which the density functions become zero. The structures were made by randomly adding one layer or "generation" at a time to a growing collection of spheres, starting from a single core sphere, and building up a tree graph.

All the spheres in a given layer $n$ have the same radius $r(n)$. The distance bond_r $r(n)$ between the centre of a new sphere and its parent in the previous layer is a fixed multiple of the sum of their radii. The direction from a parent to a new child sphere is chosen randomly on a truncated unit sphere. The truncation removes those directions with $\mathrm{z}$ component below $z l o w$, in order to cause an upward tendency in the growth.

The maximum number of spheres in a layer $n$ is a predefined constant max_sphere $(n)$. Parents and directions are chosen randomly, and the resulting new sphere is tested for collision and shape criteria, as described below, and rejected if it fails. The trials continue until max_sphere $(n)$ new spheres have been accepted, or the number of trials exceeds maxtrials $=$ tryfac $*$ max_sphere $(n)$, for a chosen factor tryfac.

The square of the distance between each trial sphere and every accepted sphere is tested against one of two collision criteria. The choice between them is determined by a generation count $g$. If the trial sphere and the test sphere have a common ancestor back at most $g$ generations (e.g., a common grandparent), then a smaller, more lenient collision factor "adjacent" is multiplied by the sum of their radii to determine the distance of closest approach. This factor may be chosen so that the contour surface connects the two spheres into one blob. If there is no close enough common ancestor, then a larger factor "nonadjacent" is used, which makes the two spheres of non-zero density disjoint, and causes the blobs to separate. 
At each level there are two other shape constraints for the sphere centres; a minimum distance from the central root node, and a minimum $z$ value. These can be used to force the coral to grow outward and/or upward.

In the current system, the minimum distance and minimum $\mathrm{z}$ limits are incremented at each level $n$ by fixed multiples of bond_r(n), so only the multipliers need be specified. In turn, bond_r $r(n)$ depends on the sphere radius $r(n)$, which is taken to be of the form $a * b^{n}$, so only a few constants need be specified to generate a coral shape. These are listed in Table 1.

1 The total number of levels in the tree

2 The initial radius, $a$, for the root sphere

3 The radius decrease pur level, $b$, with $b<1$, so that $r(n)=b * r(n-1)$

4 The bondfactor, so 'hat bond_r $r(n)=$ bondfactor $*(r(n)+r(n-1))$

5 The number $g$ of generations back for which a common ancestor allows a lenient approach distance.

6 The factor "adjacent" which multiplies $r(n)+r(i)$ to give the lenient distance for closest approach of two spheres at levels $i$ and $n$ with a common ancestor at level $\max (0, n-g, i-g)$.

7 The corresponding factor "nonadjacent" for more distantly related spheres.

8 The multiple of bond_r $r(n)$ used to increment the minimum distance from the root node.

9 The multiple of bond_r $r(n)$ used to increment the minimum $\mathrm{z}$ value.

10 The truncation level zlow for the unit sphere of directions between parent and child.

11 The base $d$ in the formula max_sphere $(n)=$ floor $\left(c * d^{n}\right)$. (c is always 2)

12 The factor tryfac in the formula maxtrials $=$ tryfac $*$ max_sphere $(n)$ for the number of random spheres to try at level $n$ before giving up.

13 An initial seed for the random number generator, used to vary the shape even when the above 12 constants are the same.

\section{Table 1}

There are two other features which can be turned on or off. The first interpolates an extra sphere between a parent $A$ and child $B$, in order to keep the density high when the distance between parent and child spheres is large. We placed this sphere to make the branches curve smoouhly. The second feature attempts to continue the curve of this branch from child $B$ to grandchild $C$, in the case that $B$ has no other children. The position of $\mathbf{C}$ is originally generated in the random search, and this random position is only revised if the smooth continuation does not violate the collision constraints. 
By changing the 13 constants in Table 1 and deciding whether to enable the above two features, a large variety of coral shapes can be generated. Initially these shapes were examined in hidden line sketches of the spheres, and only the pleasing ones were used to generate soft contour surfaces. The sketches were generated using a fraction of the outer radii for the spheres, chosen to approximate the eventual soft contour surface.

\section{Enhanced Volume Textures}

We tried to generate shapes and textures for the coral to approximate those in the photographs in Wood [15] and Kaplan [16]. According to Wood [15] (page 18) the coral polyps lay down a skeleton with a cylindrical wall (theca) surrounding the chalice which contains the digestive system of the polyp. Radial walls called septa divide the chalice, and extend outward beyond the theca towards neighbouring polyps.

We wanted to show the chalices as a round depressions, uniformly distributed on surface of the coral. A straightforward method to give the locations for these depressions is to intersect the contour surface with a collection of spheres. We chose a cubic close packing of spheres for its uniform density and because it was easy to determine the closest sphere centre to a given 3-D point. This packing is based on a regular cubic lattice, with a sphere at the midpoint of each cube edge, and also one at the centre of each cube. Each sphere is surrounded by 12 other equidistant spheres.

Figure 1 shows the result of intersecting an ellipsoid with a close packed collection of spheres. Note that the approximate circles in which the ellipsoid intersects the sphere have differing radii.

We wanted all the chalices to have about the same radius. In order to achieve this, we used an "enhanced volume texture" which is a function of both the 3$\mathrm{D}$ coordinate on the contour surface, and the surface normal at that point.

Figure 2 shows a contour surface $S$ passing near two sphere centres $C_{1}$ and $C_{2}$. Points $P_{i}, i=1,2$ are the closest points on $S$ to $C_{i}$, so that the normals $N_{i}$ lie along the lines $C_{i} P_{i}$. Then right circular cylinders of radii $r$ and axes $C_{i} P_{i}$ intersect the surface in approximate circles of radius $r$, so using these cylinders will make the circles have equal radii.

For any point $P$ in the surface $S$, we could find the nearest centre $C_{i}$ of the cubic close packing, find the distance $s$ to the corresponding axis $C_{i} P_{i}\left(C_{2} P_{2}\right.$ in 
Figure 2), and calculate the texture as a function of $s$. However, finding the points $P_{i}$ would involve a lot of iterative calculation, and also storage unless the calculation is repeated for each texture evaluation. Instead we draw a line $C_{i} D_{i}$ through $C_{i}$ parallel to the unit normal $N$ at $P$, and take $q$ as the distance from $P$ to this line. If the principle radii of curvature of the surface $S$ are large compared to the distance between the centres $C_{i}, q$ is a good approximation to $s$. Thus, we find the centre $C_{i}$ of the close packing which is nearest to $P$, and compute

$$
q=\left|\left(P-C_{i}\right)-\left(\left(P-C_{i}\right) \cdot N\right) N\right|
$$

The texture is then a function of $q$. Figure 3 shows the approximate circles coloured white whenever $q<r$. Note that the circles have approximately the same radius, but that occasionally two circles intersect at the plane of transition between two different closest centres $P_{i}$ and $P_{j}$. (This intersection can be interpreted in our application as polyp budding (see [15]).)

Figure 4 shows an ellipsoid with a bump mapped coral texture. The surface normal to the ellipsoid was perturbed radially as a function of $q$, to indicate the raised wall (theca) of the chalice and the depression it surrounds. In addition, the normal was perturbed tangentially to represent the septa which divide the chalice into sections, and also extend between them. This tangential perturbation was computed as a function of the direction of the vector TP in Figure 2. The areas where the septa cross the theca were made whiter to indicate the tentacles, by changing the surface shade.

\section{Results}

Figure 5 shows a single coral, rendered with bump mapped texture as described above. The first twelve shape parameters listed in table 1 were set respectively as $8,2 ., .9,1.6,2, .2,1.12, .67, .55,-1 ., 1.34$, and 16 ., and the sphere and smooth continuation features were used. Figure 6 shows two corals with the same texture, positioned in a sandy bottom, and rendered with shadows. The orange coral on the left is the same as the one in Figure 5. The blue-green coral on the right had shape parameters $11,2 ., .85, .7,4, .2, .7, .47, .45,0 ., 1.34$, and 30 ., and did not use the two features.

Figure 7 shows two more corals without shadows. The red one on the left had parameters $11,1.6, .85,1.67,2, .35,1.16, .6, .67, .22,1.26$, and 26. , and used the two features. The volume texture scale was set large enough to be visible, 
which made the polyp distribution at the tips of the thin branches unreasonably sparse. The green coral on the right had parameters of $8,2.6, .92, .52,5$, $.1, .35, .37, .22,-.2,1.34$ and 30 ., and did not use the two features. For positioning the raised wall of the theca in the texture, we did not use a function of $q$, as described in section 3 . We instead used a function of the ratio of the distances of a surface point to the two nearest cubic close packing sphere centres. This caused the walls to butt against each other. Figure 8 is an enlargement of the centre of Figure 7, showing the textures more clearly.

\section{Acknowledgements}

This work was performed under the auspices of the U.S. Department of Energy under contract number W-7405-Eng-48 to the Lawrence Livermore National Laboratory. We are also indebted to the University of Otago William Evans Fund for financial support and to Television New Zealand Limited for use of equipment. Carol Smith adapted the soft object ray tracing algorithm described in Wyvill and Trotman [3] to accept spheres of varying radii and proofread the manuscript. Paul Sharp helped with the production of the photographs. Kaye Saunders and Debbie Streets did the typing.

\section{Referenices}

[1] Wyvill, G., Wyvill, B. and McPheeters, C. Data Structure for Soft Objects. The Visual Computer, Vol 2, pp 227-234.

[2] Kunii, T. L. and Wyvill, G. A Simple but Systematic CSG System. Proceedings of Graphics Interface '85, pp 329-336.

[3] Wyvill, G. and Trotman, A. Ray-tracing Soft Objects. CG International '90 (T.S. Chua and T.L. Kunii eds) Springer Verlag, Tokyo (1990) pp 469-475.

[4] Bloomenthal, Jules. Modeling the Mighty Maple. Computer Graphics, Vol 19, No 3 (1985; Siggraph '85 Proceedings) pp 305-311.

[5] Aono, M. and Kunii, T.L. Botanical Tree Image Generation. IEEE Computer Graphics and Applications, Vol 4, No 5 (1984) pp 10-34.

[6] De Reffye, P., Edelin, C., Francon, J., Jaeger, M. and Peuch, C. Plant Models Faithful to Botanical Structures and Development. Computer Graphics, Vol 22, No 4 (1988; Siggraph '88 Proceedings) pp 151-158. 
[7] Viennot, X. G., Eyrolles, G., Janey, N. and Arquès, D. Combinatorial Analysis of Ramified Patterns and Combinatorial Imagery of Trees. Computer Graphics, Vol 23, No 3 (1989, Siggraph '89 Proceedings) pp 31-40.

[8] Prusinkiewicz, P. \& Lindenmayer, A. The Algorithmic Beauty of Plants. Springer Verlag, New York (1990).

[9] Kawa guchi, Y. A Morphological Study of the Form of Nature. Computer Graphics Vol 16, No 3 (1982, Siggraph '82 Proceedings) pp 223-232, and numerous subsequent computer animated films.

[10] Nishimura, H., Hirai, M., Kawai, T., Kawata, T., Shirakawa, I., \& Omura, K. Object Modeling by Distribution Function and a Method of Image Generation, Journal Electronics Communication Conference (1985) Vol J68-D, No 4 (in Japanese). English translation in Kesson, M.A. An Investigation into the Modelling of Iso-Surfaces of Scalar Fields. MA thesis, Middlesex Polstechnic (1989).

[11] Blinn, J. Simulation of Wrinkled Surfaces. Computer Graphics, Vol 12, No 3 (1978, Siggraph '78 Proceedings) pp 286-292.

[12] Peachey, D. Solid Texturing of Complex Surfaces. Computer Graphics, Vol 19, No 3 (1985, Siggraph '85 Proceedings) pp 279-286.

[13] Perlin, K. An Image Synthesizer. Computer Graphics, Vol 19, No 3 (1985, Siggraph '85 Proceedings) pp 287-296.

[14] Wyvill, G., Wyvill, B. \& McPheeters, C. Solid Texturing of Soft Objects. IEEE Computer Graphics and Applications, Vol 7, No 12 (1987) pp 2026.

[15] Wood, E. M. Corals of the World. T.F.H. Publications Inc. Ltd, Hong Kone (1983).

[16] Kaplan, E. H. A Field Guide to Coral Reefs. Houghton Mifflin Company, Boston (1982). 


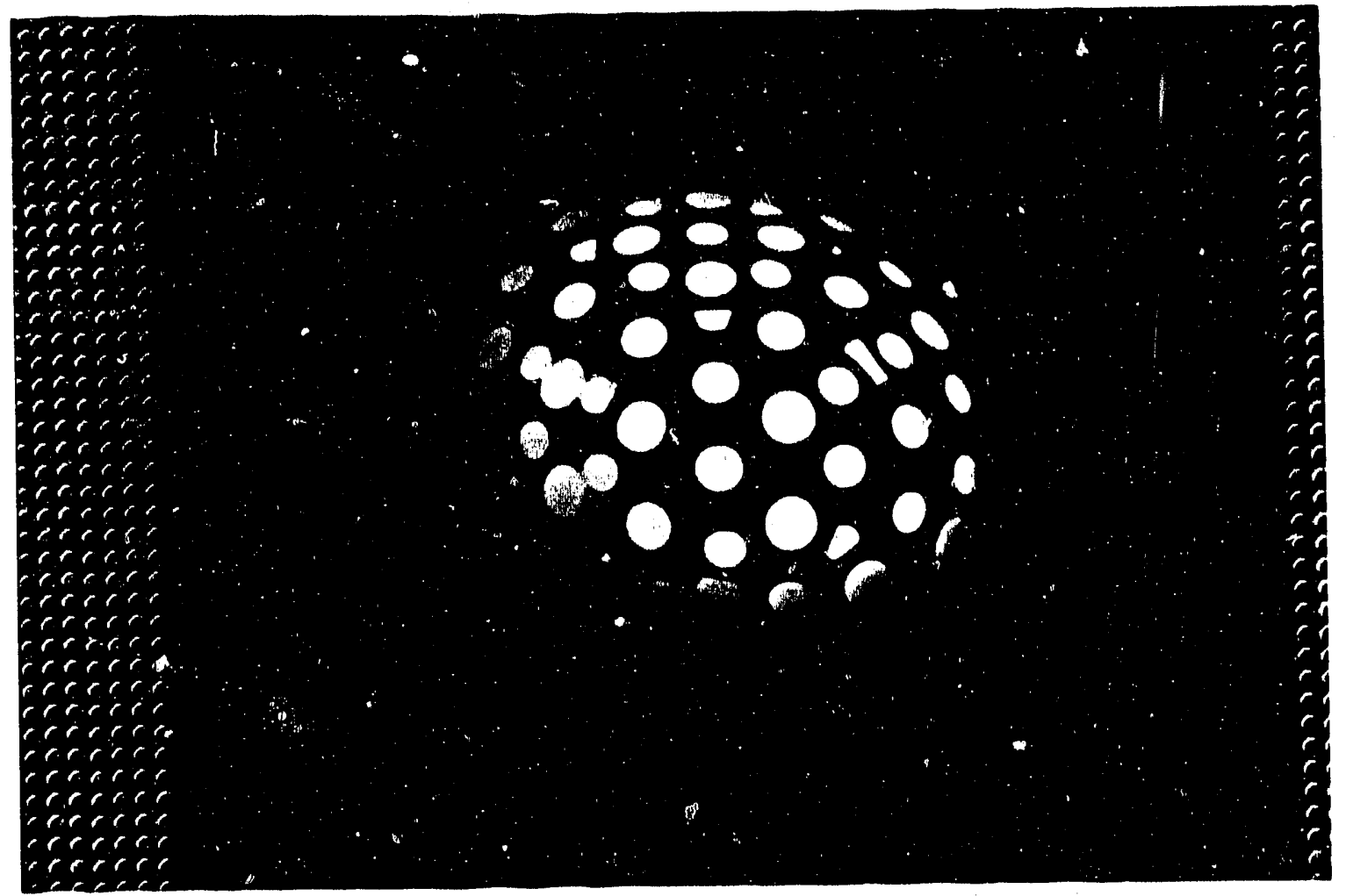

Fipire 3
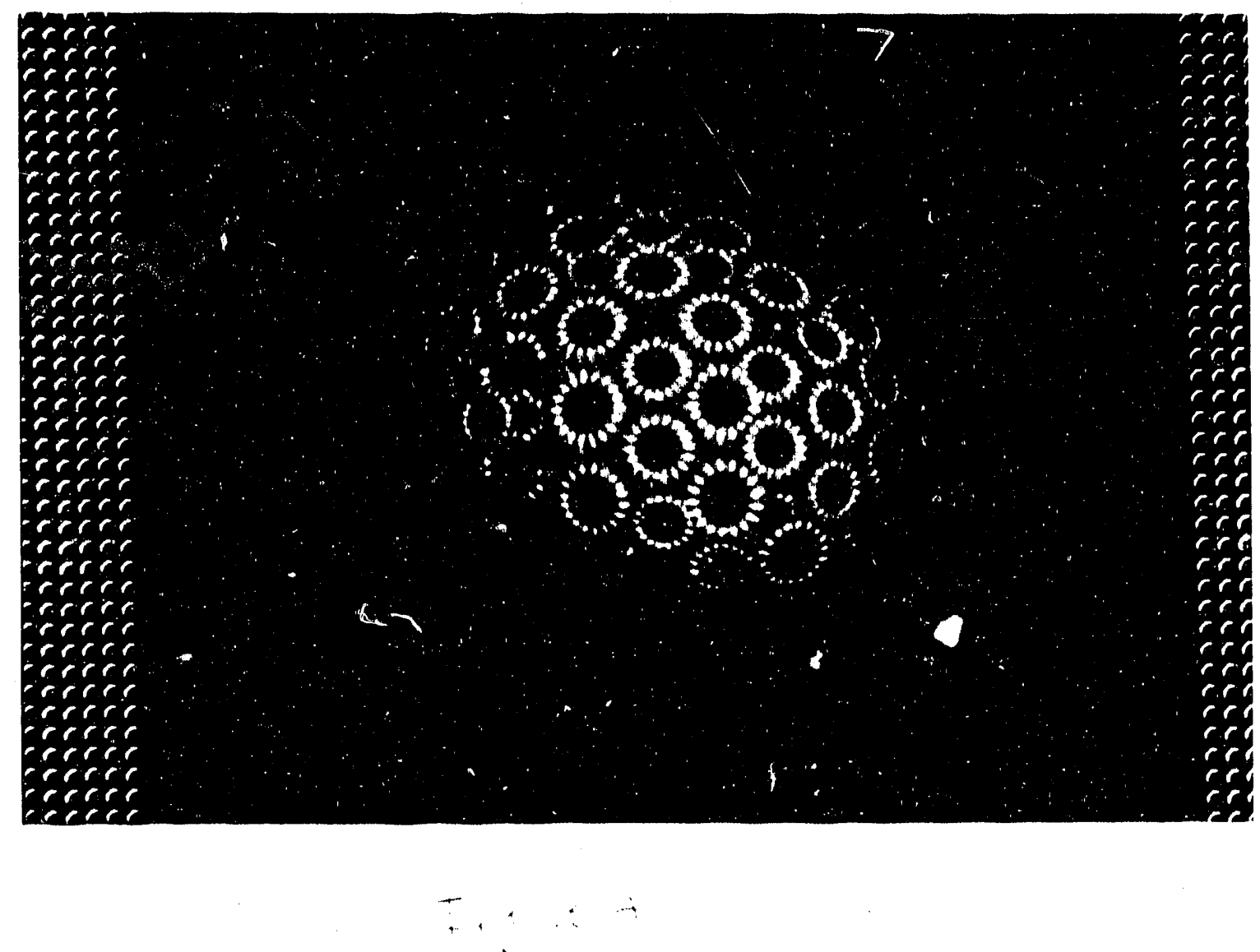


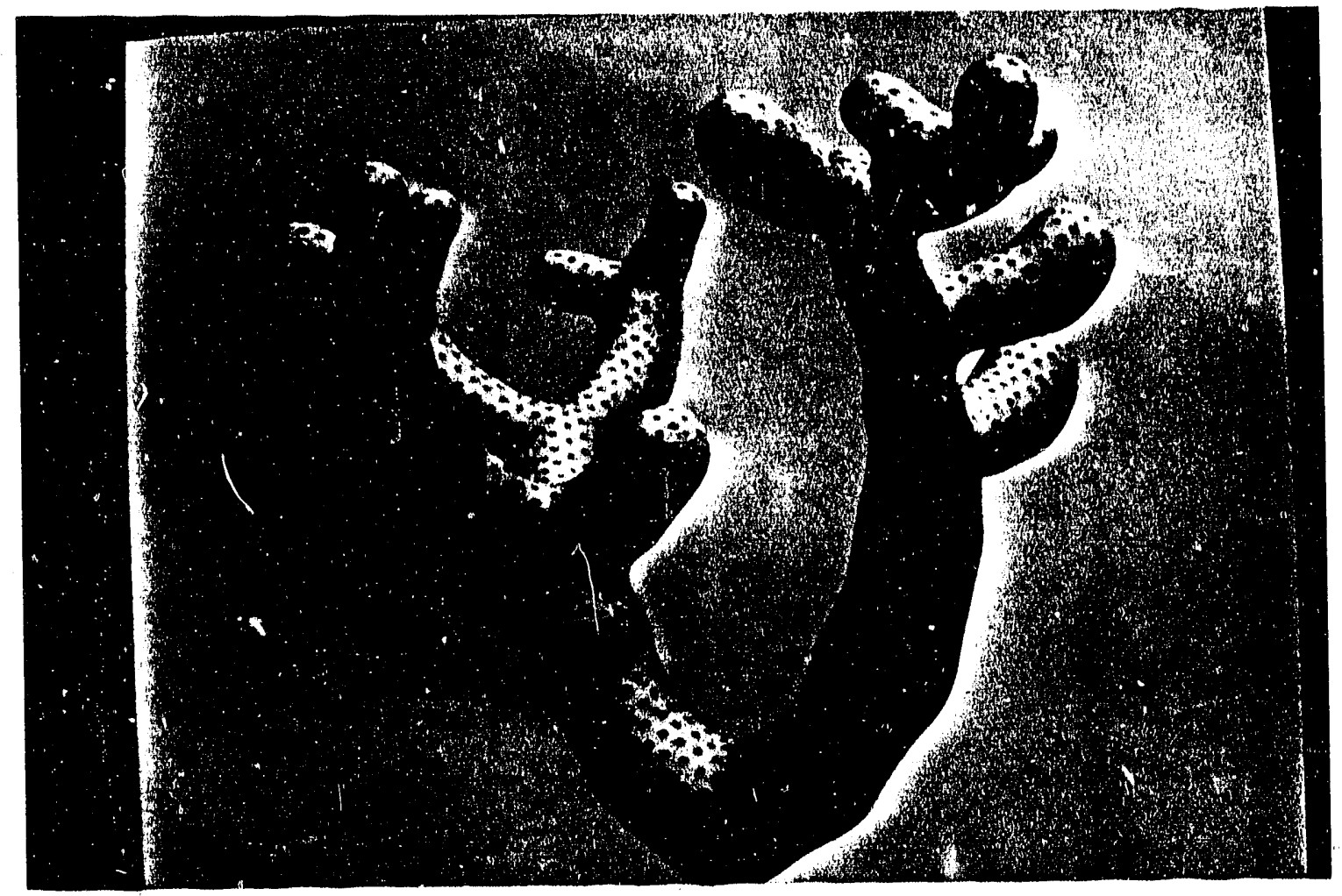

$F$

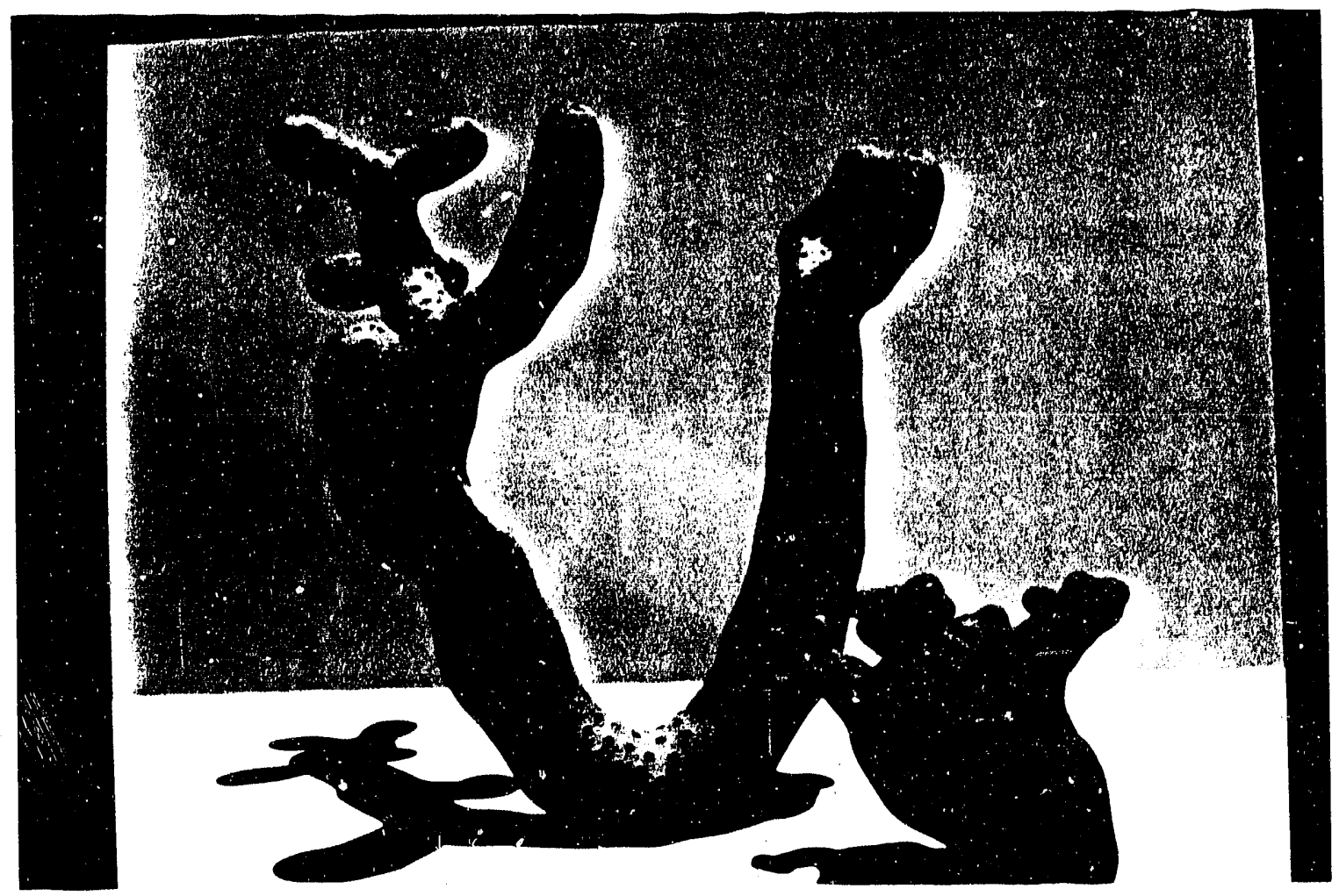



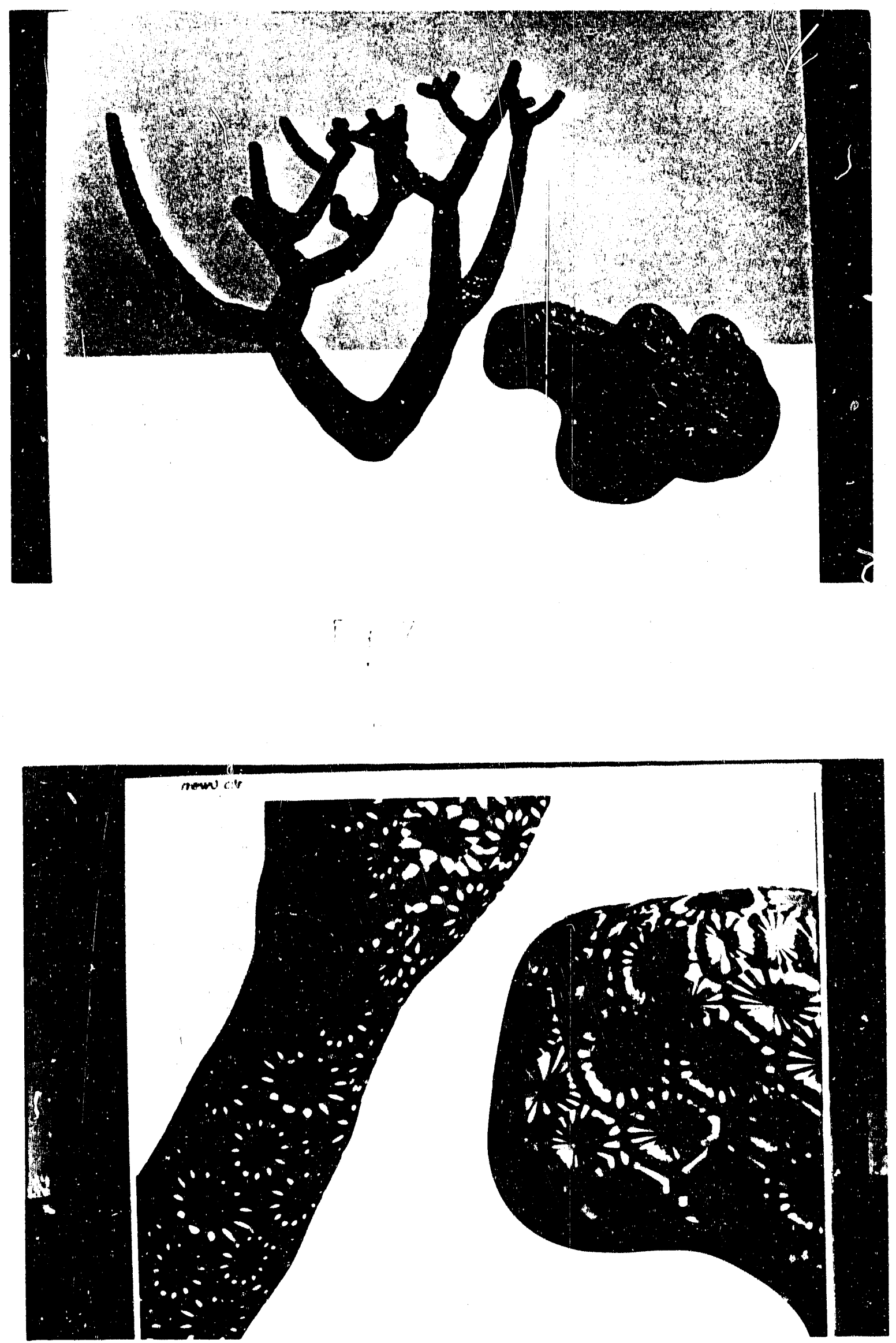

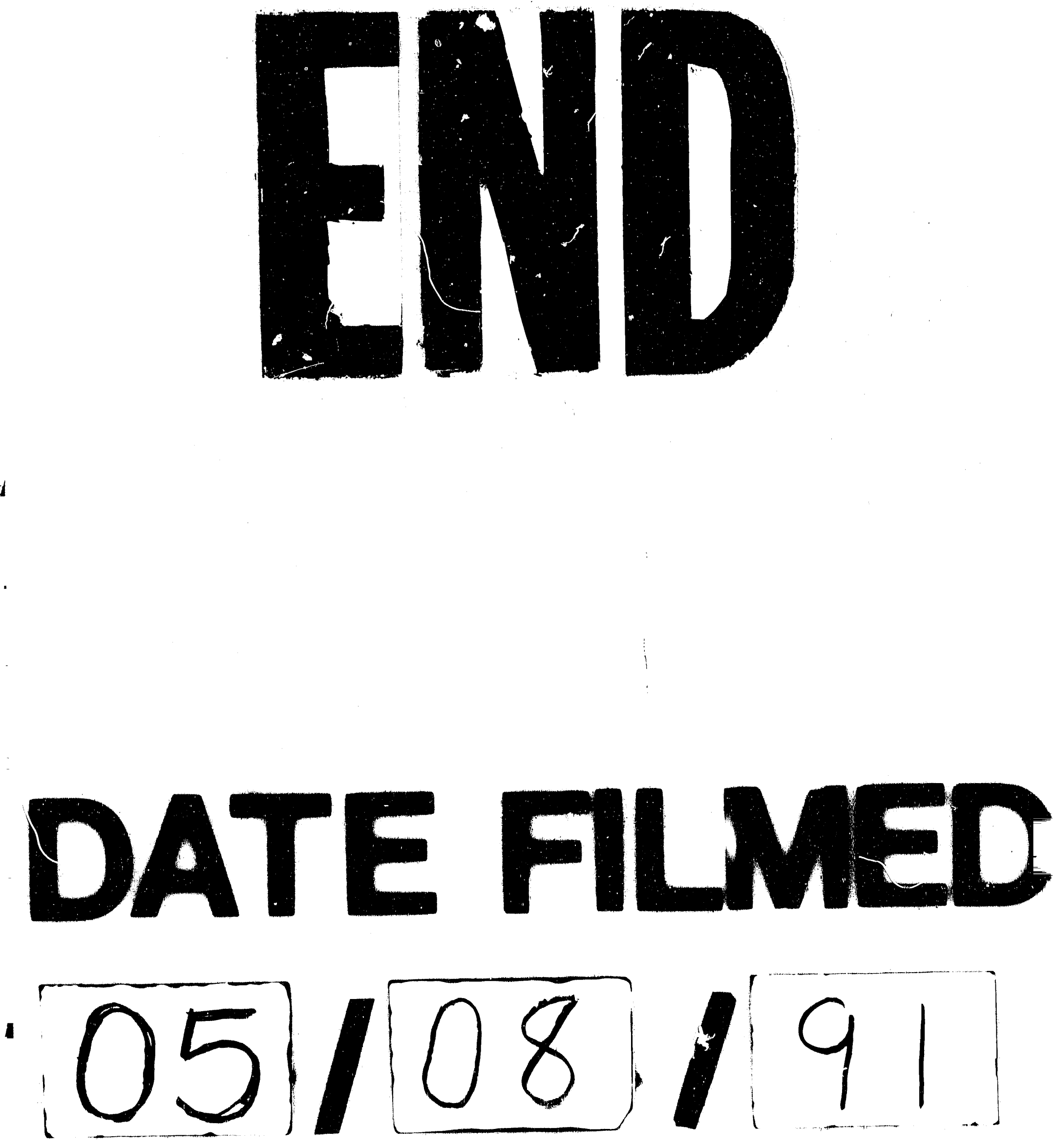
